\title{
Directing attention externally enhances agility performance: a qualitative and quantitative analysis of the efficacy of using verbal instructions to focus attention
}

\author{
Jared M. Porter ${ }^{*}$, Russell P. Nolan ${ }^{1}$, Erik J. Ostrowski ${ }^{1}$ and Gabriele Wulf ${ }^{2}$ \\ Department of Kinesiology, Southern Illinois University Carbondale, Carbondale, IL, USA \\ 2 Department of Kinesiology and Nutrition Sciences, University of Nevada, Las Vegas, NV, USA
}

\author{
Edited by: \\ Martyn Standage, University of Bath, \\ UK \\ Reviewed by: \\ Norbert Hagemann, University of \\ Kassel, Germany \\ Marco Taubert, Max-Planck-Institute for \\ Human Cognitive and Brain Sciences, \\ Germany \\ James Hardy, Bangor University, UK \\ *Correspondence: \\ Jared M. Porter, Department of \\ Kinesiology, Southern Illinois University \\ Carbondale, Davies Hall - Mail Code \\ 4310, 1075 South Normal Avenue, \\ Carbondale, IL 62901, USA. \\ e-mail: jporter@siu.edu
}

The primary purpose of this study was to investigate if focusing attention externally produced faster movement times compared to instructions that focused attention internally or a control set of instructions that did not explicitly focus attention when performing an agility task. A second purpose of the study was to measure participants' focus of attention during practice by use of a questionnaire. Participants $(N=20)$ completed 15 trials of an agility " $L$ " run following instructions designed to induce an external (EXT), internal (INT) attentional focus or a control (CON) set of instructions inducing no specific focus of attention. Analysis revealed when participants followed the EXT instructions they had significantly faster movement times compared to when they followed the INT and CON set of instructions; consistent with previous research the INT and CON movement times were not significantly different from each other. Qualitative data showed when participants were in the external condition they focused externally $67 \%$ of the time. When they were in the internal condition they focused internally $76 \%$ of the time, and when they were in the control condition they did not use an internal or external focus of attention $77 \%$ of the time. Qualitative data also revealed participants in the EXT, INT, and CON conditions switched their focus of attention at a frequency of 27,35 , and $51 \%$ respectively.

Keywords: skill assessment, directions, practice

\section{INTRODUCTION}

For more than a decade findings reported in motor learning literature have consistently demonstrated that providing instructions that focus a learner's attention externally, rather than internally, generally result in enhanced motor skill performance (for a review, see Wulf, 2007). According to Wulf et al. (1998) an external focus is characterized by a learner directing their attention to the effects that their movements have on the environment. This is in contrast to focusing on specific body parts or movements themselves, which constitutes an internal focusing of attention. For example, a baseball coach who desires to increase the velocity of a thrown ball may instruct a pitcher to focus on increasing the speed of the baseball as they progress through their pitching windup and release. Providing this type of instruction would induce an external focus because the athlete is asked to focus on the speed of the ball. Another baseball coach desiring the same outcome may instruct the pitcher to focus on increasing the speed of their pitching arm during the windup and release. This type of instruction would induce an internal focus of attention because the athlete is asked to focus on the movement of their arm during the pitching motion.

Many reported experiments have demonstrated beneficial effects of using an external focus of attention in a variety of movementrelated contexts, especially when performing motor skills that require a need for the accurate placement of an object. For example, studies have shown that golf shot accuracy can be improved by focusing attention externally rather than internally (Wulf and Su, 2007; Bell and Hardy, 2009). Similar methods have been used to improve basketball shot accuracy (Al-Abood et al., 2002), volleyball serve placement (Wulf et al., 2002, Exp. 1), and soccer shot precision (Wulf et al., 2002, Exp. 2). The benefits of using an external focus are not limited to skills that require the accurate placement of an object. Studies have demonstrated that providing instructions or feedback that prompt individuals to focus their attention externally rather than internally improves balance (Shea and Wulf, 1999), vertical jump (e.g., Wulf et al., 2007), and standing long jump performance (Porter et al., 2010).

The constrained action hypothesis is commonly used to explain why focusing attention externally, rather than internally or using no specific focus, facilitates the learning of motor skills (see Wulf et al., 2001). This hypothesis suggests that focusing on the effects (i.e., external focus) of a movement allows motor behaviors to happen automatically or with unconscious motor control. However, when individuals focus on the movements of their body (i.e., internal focus) they interfere with the automatic processes of motor behavior. The interference that results from this conscious control of the motor control system results in decreased accuracy, slower movements, and overall depressed motor performance (Wulf, 2007). Consistent with the perspectives of the constrained action hypothesis, two studies (Vance et al., 2004; Marchant et al., 2009) using electromyography (EMG), demonstrated that using an external focus of attention during a bicep curl task resulted in a more efficient contraction of not only the agonist (i.e., the biceps muscle) but also the antagonist (i.e., the triceps muscle). This suggests that simply directing attention externally during motor skill execution causes the motor control system to recruit muscle fibers more efficiently possibly resulting in a more highly organized motor program. 
It is interesting to note that experimental results show participants not receiving a specific set of focus-directing instructions tend to perform similarly to participants who receive instructions or feedback inducing an internal focus (e.g., McNevin and Wulf, 2002; Wulf and $\mathrm{Su}, 2007)$. More specifically, participants in these "control" conditions are not provided instructions or feedback designed to induce an internal or external focus of attention, rather the participant is allowed to focus their attention using their own discretion. These findings suggest when participants are allowed to choose their own focus of attention they may use an internal focus, or other type of focus that interferes with automatic processing resulting in depressed skill performance (Wulf, 2007). The assumption that learners may focus their attention internally when allowed to choose is speculative to date, as few studies have attempted to measure what is focused on when participants are practicing under "control" conditions. In fact, little attempt has been made to measure the accuracy in which attention is directed following verbal instructions and feedback that are designed to induce either an internal or external focus of attention (Gray, 2004; Poolton et al., 2007; Lawrence et al., in press). The studies that have attempted to measure the accuracy of attentional directing have commonly used a dual task methodology (Beilock et al., 2002; Gray, 2004; Castandeda and Gray, 2007), which creates an unnatural performance environment, consequently reducing the applicability of the experimental findings. It is not our goal in this paper to discredit the work conducted by Beilock and colleagues, or Gray and colleagues; rather it is our goal to better understand and expand the generalizations of how subtle word changes in verbal instructions influence focus of attention and task execution when performing a complex motor skill.

Numerous studies have demonstrated the performance benefits of using an external focus of attention when the action goal requires the accurate placement of an object (e.g., Al-Abood et al., 2002; Wulf et al., 2002; Wulf and Su, 2007). Fewer studies have demonstrated that using an external, rather than internal, focus of attention can also improve the performance of motor skills that require a whole body movement, such as jumping (Wulf et al., 2007; Porter et al., 2010). Some questions remain about the generalizability of these findings in relation to complex motor skills that require locomotion (Raab, 2007), or tasks that require a person to quickly change the direction of their entire body during the performance of a skill (Porter et al., 2010). Tasks involving agility are commonly used in sport, educational, and rehabilitative settings to evaluate performance and motor skill development (Young, and Farrow, 2006; Bennett, 2008; Sayers et al., 2008; Spaniol, 2009). Agility is defined as the ability to change the direction of the body rapidly using a combination of strength, speed, balance, and coordination (Sayers et al., 2008). In addition, agility is a complex whole body movement that is frequently linked to being a skillful mover (Young, and Farrow, 2006; Bennett, 2008; Sayers et al., 2008; Spaniol, 2009). Studies have shown that vertical jump (Wulf et al., 2007) and standing long jump (Porter et al., 2010) performance are influenced by attentional focus, therefore it is hypothesized that a complex whole body movement requiring agility would also be influenced by manipulating the performer's focus of attention.

Thus, the primary purpose of this study was to investigate if changing a performer's focus of attention influences the performance of a task requiring agility. It was predicted that participants completing an agility task would have a faster movement time when following instructions designed to induce an external rather than internal or no focus of attention. The secondary purpose of the current study was to incorporate a manipulation check into the experimental design to measure the accuracy and consistency by which participants followed the prescribed attentional focus instructions. Another goal of incorporating a manipulation check into the experimental design was to gain a level of understanding regarding what participants focused on when performing with a neutral set of instructions (i.e., control condition), which were designed to induce no specific focus of attention.

\section{MATERIALS AND METHODS PARTICIPANTS}

Undergraduate college students $(N=20 ; 14$ males, 6 females; mean age $=21.15$ years, $\mathrm{SD}=2.16$; mean height $=175.9 \mathrm{~cm}, \mathrm{SD}=11.33$; mean weight $=79.34 \mathrm{~kg}, \mathrm{SD}=13.43$ ) were recruited to participate in this study. Participants were not currently or formerly a member of a professional or semi-professional athletic team. In addition, participants agreed they had not received formal sprint or agility training. Although participants were considered untrained, we do acknowledge that participants likely performed tasks requiring agility at some point in their lives and were likely involved in a variation of agility testing in a physical education class or through involvements in youth/recreational sports. All participants agreed they had no memory of previously performing the specific agility test that was used in the current study. All participants signed an informed consent and filled out a medical history form before participating in the study. All forms were approved by the University's Institutional Review Board. Participants were naive to the purpose of the study.

\section{APPARATUS AND TASK}

The agility course was set up on a hard wood surface in a basketball gymnasium at the University. The agility "L" test (Webb and Lander, 1983; Gabbett, 2006; Gabbett et al., 2008) consists of two 5-m sections connected at a right angle to make an "L" shape (see Figure 1). Previous studies using the agility L run have reported an intraclass correlation coefficient of 0.90 or higher, suggesting the test is reliable. Total movement time was measured using infrared timing gates (Brower Timing Systems ${ }^{\circledR}$, model IRD-T175) located at the start and finish line. Movement time began when participants crossed the infrared beam at the start line and stopped when participants crossed the same infrared beam at the finish line. After each trial, the movement time was entered into a computer spreadsheet and saved for later analysis. Each of the 20 participants completed a total of five agility runs in each of the three conditions, equaling a total of 100 practice trials per condition.

\section{PROCEDURES}

Using a within participant design, all participants completed five maximum effort trials under each focus condition (Control, External, and Internal). Participants read the prescribed set of instructions prior to each trial. They were then asked by the experimenter to repeat the instructions. If a participant could not accurately repeat them, he or she was asked to re-read the 
instructions. Control (CON) instructions were to "run through the course as quickly as you can with maximum effort." External (EXT) instructions were to "Run through the course as quickly as you can with maximum effort. This agility test consists of two parts, a running component and a turning component. For each running component, I want you to focus on running toward the cone as rapidly as possible. For the turning component, I want you to focus on pushing off the ground as forcefully as possible." Internal (INT) instructions were to "Run through the course as quickly as you can with maximum effort. This agility test consists of two parts, a running component and a turning component. For each running component, I want you to focus on moving your legs as rapidly as possible. For the turning component, I want you to focus on planting your foot as firmly as possible."

Participants completed one experimental condition per day for a total of 3 days. They completed a total of five practice trials each day following the prescribed attentional focus instructions. Each set of trials for each condition was performed on separate, nonconsecutive days of the week (i.e., Monday, Wednesday, and Friday) at the same time each day. Pilot testing indicated when participants performed in the CON condition on a day following either the EXT or INT condition they tended to use the prior day's focus of attention while performing in the CON condition. Because of this unwanted influence, all participants in the current study performed the CON condition on day 1 ; the order of the INT or EXT condition was counterbalanced across days 2 and 3 for all participants to control for order affects.

Prior to data collection each day, participants completed a $5 \mathrm{~min}$ dynamic warm-up by briskly walking laps around the basketball gymnasium where the experimental testing took place. After the warm-up, participants saw a diagram (see Figure 1) and read a written description of the agility course. The researcher then walked the participant through the agility course for further understanding. This walk-through took place silently so the researcher would not inadvertently influence the attentional focus of the participant, or indirectly offer a strategy that could be used by the participant to improve performance. The walk-through was followed by a



FIGURE 1 | Agility “L” test. Participant begins at start line beside cone A. Run toward cone $\mathrm{B}$, pivot around cone $\mathrm{B}$ and run toward cone $\mathrm{C}$. Run around cone $\mathrm{C}$ and run back toward cone $\mathrm{B}$. Pivot around cone $\mathrm{B}$ and run across finish line. seated 2 min rest where the participant read the prescribed focus instructions for the upcoming set of trials. After the rest period, the participant was instructed to stand at the start line where he or she was asked to repeat the focus instructions for that trial. Participants were then instructed to start when ready. After the trial, each participant sat for another 2 min rest where they could reexamine the diagram and instructions of the course as well as the prescribed focus of attention instructions. While participants were seated during this 2 min rest they were also asked to write a response to the following question: "What were you focusing on during the previous trial? If you did not focus on anything particular during the trial, please leave the question blank." Each written response was collected by the experimenter prior to the start of the next trial run. At no time were participants allowed to view a previously written response. This procedure continued until all five trials were completed each day. Participants were not informed of their movement time or provided any performancerelated feedback.

\section{DATA ANALYSIS}

Movement time values were analyzed using a 3 (focus of attention: CON, EXT, INT) $\times 5$ (trial) repeated measures analysis of variance (ANOVA). Cohen's effect size (ES) statistics (Cohen's d) were calculated to determine the magnitude of observed significant post hoc performance differences. Effect sizes were based on the criteria of $d<0.30$, small; $d=0.31-0.70$, moderate; and $d>0.71$, large.

\section{RESULTS \\ MOVEMENT TIME}

Data were analyzed using the Statistical Package for the Social Sciences (SPSS) Version 16. Movement times under the three focus conditions are presented in Figure 2. As can be seen, movement times were faster in the EXT condition. The main effect for focus of attention was significant, $F(2,285)=5.089, p<0.01$. A Tukey HSD post hoc analysis of the Focus of Attention main effect indicated that the $\operatorname{EXT}(M=6.10, \mathrm{SD}=0.14)$ condition was significantly faster than both the INT $(p<0.01)(M=6.45, \mathrm{SD}=0.12)(\mathrm{ES}=0.23)$ and $\operatorname{CON}(p<0.04)(M=6.36, \mathrm{SD}=0.14)(\mathrm{ES}=0.19)$ conditions. This analysis also indicated that the $\mathrm{CON}$ and INT groups were not



FIGURE 2 |Agility run times for the external (EXT), internal (INT), and control (CON) conditions. 
significantly different $(p<0.66)$. A main effect was not observed for trial, $F(4,285)<1$. The Focus of Attention $\mathrm{x}$ Trial interaction was not significant, $F(8,285)<1$.

\section{MANIPULATION CHECK}

Questionnaire responses were organized and coded collectively by the authors within each of the experimental conditions into one of three broad categories: Internal, External, or Other. The first, second, and third authors discussed all written responses and agreed how each response should be coded. In addition, the first and fourth author further discussed less clear responses and collectively agreed on the category assignment. The authors then further coded the responses into subcategories within each broad category (see Figure 3). If participants provided a written response indicating that their attention was solely focused internally, then that response was placed into the "Internal" category. For example, three different participants provided the following questionnaire responses: "I focused on moving my legs as quickly as possible while planting my feet firmly on the ground while turning"; "Moving my legs as rapidly as I could"; "I focused on what foot to pivot with to give the best advantage possible." We determined that these responses indicated that the participants were exclusively focusing their attention internally because they reported attending to their legs and/or feet.

This is in contrast to participants who provided responses indicating that they focused their attention externally. For example, three different participants stated they focused on "Running toward the cone as rapidly as possible and pushing off the ground as forcefully as possible"; "I really focused on cutting the corners close and pushing off the ground forcefully"; or "Getting to the cone as quickly as possible." These responses suggest that participants recalled only using an external focus as they directed their attention solely to the result of the movement or features in the environment (i.e., cutting around the cone or pushing off the ground). Thus, these responses were placed into the "External" category.

At times participants provided responses indicating they were not directing their attention exclusively internally or externally, and at times they reported not attending to anything particular. For example, some participants left the questionnaire blank, possibly indicating they did not consciously focus their attention or could not explicitly recall what they attended to on the previous trial. This observation will be elaborated on in the Section "Discussion." In addition to leaving the questionnaire blank some participants reported they used a combination of an internal and external focus of attention while performing the agility task. For example, three different participants stated "I focused on moving my feet quickly and staying close the cones"; "Keeping my body low while turning around the cones"; and "I focused on my foot work in order to quickly and successfully move around the cones. We concluded that these types of responses indicated the participants used a mixture of internal (i.e., feet) and external (i.e., moving around the cones) focus of attention while executing the previous trial. It was also common for participants to report they focused on issues related to time. For instance, three different participants reported "I focused on going as fast as possible"; "Performing faster than the previous run"; and "Running through the course as quick as

\begin{tabular}{|c|c|c|c|c|c|c|}
\hline \multicolumn{7}{|l|}{ INT Condition } \\
\hline Broad Category: & External & & Internal & & & Other \\
\hline Total Responses: & 1 & & 76 & & & 23 \\
\hline Subcategory: & Floor & & Feet Legs & Legs \& Feet & & Time $\underline{\text { Mixed Blank }}$ \\
\hline Responses: & 1 & & $30 \quad 17$ & 29 & & 13 \\
\hline \multicolumn{7}{|l|}{ EXT Condition } \\
\hline Broad Category: & External & & & Internal & & Other \\
\hline Total Responses: & 67 & & & 2 & & 31 \\
\hline Subcategory: & Floor \& Cone & $\underline{\text { Cone }}$ & Floor & Feet Legs & & Time $\underline{\text { Mixed }}$ Blank \\
\hline Responses: & 39 & 10 & 18 & 1 & & 18 \\
\hline \multicolumn{7}{|l|}{ CON Condition } \\
\hline Broad Category: & External & & Internal & & & Other \\
\hline Total Responses: & 13 & & 10 & & & 77 \\
\hline Subcategory: & Cone Floor & & Feet Legs & Legs \& Feet & Body & Time Mixed Blank \\
\hline Responses: & 6 & & 5 & 1 & 3 & 19 \\
\hline
\end{tabular}

FIGURE 3 | In-depth breakdown of questionnaire response categories. Each number represents the total number of questionnaire responses in each of the broad and subcategories for each experimental condition. The total number of responses in each subcategory equals the number listed in its corresponding broad category. Within the "Other" category the subcategory of "Time" includes the number responses that suggested the participant focused on cues related to their movement time. The subcategory of "Mixed" includes responses that included a mixture of internal and external focus. 
possible." Questionnaires that were left blank, in addition to reports that indicated a mixture of internal and external focus was used, and responses that indicated that focus was directed at improving movement time were placed into the "Other" category.

Analysis of qualitative data indicated that participants in the INT condition reported they solely focused their attention internally on $76 \%$ of the trials, and externally on $1 \%$ of the trials. This analysis also indicated that participants in the INT condition used an "other" focus of attention $23 \%$ of the time. Qualitative data also indicated that participants in the EXT condition focused their attention externally during $67 \%$ of the trials, internally on $2 \%$ of the trials, and they used an "other" focus of attention on $31 \%$ of the trials. Finally, this analysis revealed that participants in the CON condition focused their attention externally on $13 \%$ of the trials, internally $10 \%$, and used an "other" focus of attention $77 \%$ of the trials. See Figure 3 for an in-depth breakdown of questionnaire response categories.

Using the questionnaire responses we also calculated the frequency of attentional switching for each participant within each of the three experimental conditions (i.e., CON, INT, EXT). To calculate how frequently participants switched their focus we read the five written responses provided by each participant in the order they were reported. Then we compared each written report to the previous trials written report to determine if the participant changed their focus from the previous trial to the current trial. It was not uncommon for participants to switch from an internal, external, or "other" focus of attention between attempts while practicing their five trials within one experimental condition. For example, one participant in the CON condition reported on trial 2 "I focused on trying to stay close to the cones as possible when rounding them"; however on the following trial (i.e., trial 3) the same participant reported they focused on "Keeping my legs moving as fast as I could and trying to pivot my feet harder." This series of responses suggest that the participant shifted from an external focus (i.e., staying close to the cone) on trial 2 to an internal focus (i.e., moving legs and pivoting feet) on trial 3 .

At times participants reported a within condition focus of attention switch between trials of the agility task. For example, one participant in the INT condition reported on trial 3 "I was focusing on my legs moving." The same participant reported on the following trial (i.e., trial 4) "I was focusing on my feet." The same participant reported on trial 5 "I was focusing on moving my feet as rapidly as I could." These series of reports suggests the participant continually focused their attention internally on trials 3 , 4 , and 5 , as they were directed to do so in the INT conditions verbal instructions. However they reported shifted their internal focus from their legs on trial 3 to their feet on trial 4 , and continued to focus on their feet on trial 5. To determine the number of times participants switched their focus of attention we calculated the number of switches between the broad categories in addition to the attentional focus switches that took place between the subcategories as identified in Figure 3. Understanding how frequently participants choose to shift their focus may offer some additional insight why inducing an external rather than internal or no focus of attention often facilitates motor skill learning and performance.

The results of the frequency of attentional switching calculation revealed when participants were in the INT condition they switched their focus of attention on 35 of the 100 performed trials. This suggests when participants were in the INT condition they switched their focus of attention at a frequency of $35 \%$. When participants were in the EXT condition they switched their focus of attention between 27 of the 100 trials, or at a frequency of $27 \%$. When participants were in the CON condition they switched their focus of attention between 51 of the 100 trials, or at a frequency of $51 \%$.

\section{DISCUSSION}

Previous studies have shown that the benefits of using an external focus have been observed when performing a variety of motor skills (Al-Abood et al., 2002; Wulf et al., 2002; Wulf and Su, 2007; Bell and Hardy, 2009). The primary purpose of the current study was to investigate if the benefits of using an external focus of attention generalize to a task requiring agility. It was hypothesized that inducing an external focus of attention would result in faster movement times when performing an agility task compared to performances that occurred after participants read instructions that were designed to induce an internal or no specific focus of attention. The secondary purpose of this study involved the inception of a manipulation check into the experimental design to measure the accuracy that participants followed the prescribed instructions by measuring focus of attention across trials in each of the experimental conditions. An additional goal of using the manipulation check was to determine what participants focused on when they performed the task following a neutral set of instructions, as was the case in the CON condition. In addition we sought to measure the frequency participants switched their focus within each experimental condition.

The results of the present study lend support in favor of the hypothesis that providing instructions that direct a performer's attention externally facilitates agility performance compared to instructions that focus attention internally or do not explicitly focus attention. Consistent with previous studies (e.g., McNevin and Wulf, 2002; Wulf and Su, 2007), the present findings showed when participants were provided a neutral set of instructions their performances did not differ significantly from conditions that prompted an internal focus of attention. Qualitative data results indicate that the prescribed instructions did in fact induce the appropriate focus of attention 67 and $76 \%$ of the time for the EXT and INT conditions respectively. The results of the questionnaire responses further indicated that the neutral set of instructions in the CON condition achieved the goal of not inducing an explicit internal or external focus of attention $77 \%$ of the time. Generally speaking, the results of the qualitative data suggest that the prescribed instructions did indeed induce the desired focus of attention most of the time in each of the three experimental conditions. The results of the questionnaire responses also indicated when participants where in the CON condition they chose to switch their focus of attention at a frequency of 51\% compared to participant in the INT and EXT conditions which switched their focus of attention at a frequency of 35 and $27 \%$, respectively.

Although the present study used performance outcome measures (Magill, 2007) to evaluate performance, recent experiments using performance production measures (Magill, 2007) offer possible explanations as to why adopting an external focus of attention is beneficial for agility tasks. First, Wulf et al. (2007) demonstrated participants who were instructed to focus attention externally while 
performing a vertical jump produced more force than participants who directed attention internally or participants who used a neutral focus of attention. Force was not measured in the current study, however it seems plausible that participants in the EXT group may have produced greater ground reaction forces when sprinting toward and pivoting around the cones compared to participants in the INT and CON group. This increased force production may have resulted in a significantly faster movement time for the EXT condition. A second possible explanation is related to the efficiency of muscle fiber recruitment. It has been demonstrated that focusing attention externally results in a more effective movement pattern by increasing the efficiency of muscle fiber recruitment which then enhances performance (Vance et al., 2004; Zachry et al., 2005; Marchant et al., 2009; Schücker et al., 2009). Although EMG was not measured in the current study, another plausible explanation for the current observed results is that the EXT group used a more effective coordination pattern as a result of efficient muscle fiber recruitment. A more effective coordination pattern could allow performers to accelerate/decelerate and change direction more efficiently while maintaining a faster speed throughout the trial. Future experimentation using force production measures or EMG may be able to validate these speculations.

As previously mentioned, many experiments have demonstrated when participants are allowed to choose their own focus of attention or are provided a neutral set of instructions they tend to perform similarly to participants that use an internal focus of attention (e.g., McNevin and Wulf, 2002; Wulf and Su, 2007; Wulf et al., 2007). Because of this similarity, one conclusion from these results is that participants who receive a neutral set of instructions may chose to use an internal focus of attention. The results of the current study suggest that this may not be the case; in fact the current results revealed that participants in the CON condition focused internally only $10 \%$ of the time. The qualitative results further revealed that participants in the CON group used an "Other" focus of attention $77 \%$ of the time (see Figure 3). It is worth noting when participants were in the INT condition they only left the questionnaire blank on $2 \%$ of the trials. In comparison, when participants were in the EXT condition they chose to leave the questionnaire blank on $7 \%$ of the trials. However, when participants were in the CON condition they left the questionnaire blank on $22 \%$ of the trials. The higher percentage of blank questionnaire responses while practicing the task in the CON condition may indicate that more participants did not consciously attend to specific cues while performing the agility task while following a neutral set of instructions. It is also worth noting that participants in the CON reported focusing on cues related to "time" at a higher rate (i.e., $36 \%$ of trials) compared to participants in the INT condition (13\% of trials) and EXT condition (6\% of trials). Perhaps focusing on movement time is not an effective practice strategy to improve agility performance.

The results of the attentional switching calculations suggest when participants in the CON condition were provided a neutral set of instructions they chose to frequently switch their attention, perhaps in search of a task solution to improve their agility performance through the development of an efficient motor program. An additional conclusion drawn from the questionnaire responses is when participants were not explicitly instructed to focus internally or externally they chose to be very inconsistent in their selection of attentional focus, possibly focusing on different features of the environment, their movements, skill characteristics, or a combination of these factors in search of finding a method of improving their motor performance. The results of the CON condition's questionnaire responses are consistent with the Fitts and Posner (1967) and Gentile's (1972, 2000) stages of learning models, which suggest that beginners continually search the environment and the task itself for solutions to solve the movement problem in hopes of achieving the action goal. Perhaps this erratic search strategy observed by participants in the CON condition is part of the natural learning process. However, this strategy may constrain the movements and interfere with the development of automatic processing, resulting in movement productions and performance outcomes similar to participants who were directed to focus internally. This provides further evidence that practitioners should provide instructions that explicitly induce an external focus of attention. Doing this may encourage automatic processing to develop in low skilled learners or novices, which directly facilitates the improvement of motor behaviors. An interesting question that needs to be addressed in future work is whether or not highly skilled performers use this same strategy when provided a neutral set of instructions, or whether this pattern is unique to novices. It is worth noting that learning was not measured in the current study and, as indicated in the Section "Materials and Methods," we did not consider the participants of this study to be complete novices.

There are limitations to the current findings, and many of these limitations highlight the need for continued experimentation to validate the results reported here. For example, future studies should utilize a between-participant design and implement a retention and/or transfer test following practice. Doing this would indicate if the verbal instructions used in this study result in enhanced motor skill learning, or if the findings reported here are a temporary phenomena observed only after instructions are given. Future research should also test agility performance using highly skilled participants, which is critical to test the generalizability of the current findings. This point is important considering findings of a recent study (Porter et al., 2010) indicated that during practice $84.6 \%$ of professional track and field coaches provided instructions to athletes that referenced body and/or limb movements, which resulted in a majority $(69.9 \%)$ of athletes using an internal focus when competing. In addition, future research is needed to measure the validity and reliability of the manipulation check used in this experiment. This last suggestion is especially needed to better understand how participants focus their attention when they are performing in control conditions following a neutral set of instructions. Understanding what participants are focusing on when allowed to choose their focus would offer valuable insight into the motor skill learning process. In return, better understanding this process would allow practitioners to create efficient and effective practice environments.

The findings presented here add to a growing body of literature suggesting if practitioners desire to create effective instructions they should deliver those instructions in a way that directs the learner's attention externally. The results of this experiment make a unique contribution to the motor learning literature by demonstrating that a complex motor skill requiring agility can be enhanced when the 
learner's attention is directed externally. The results of this study also provide a plausible explanation why participants receiving a neutral set of verbal instructions (i.e., control condition) generally perform similarly to participants who focus their attention internally. It is common for practitioners to use agility based testing to

\section{REFERENCES}

Al-Abood, S.A., Bennett, S. J., Hernandez, F. M., Ashford, D., and Davids, K. (2002). Effects of verbal instructions and image size on visual search strategies in basketball free throw shooting. J. Sports Sci. 20, 271-278.

Beilock, S. L., Carr, T. H., MacMahon, C., and Starkes, J. L. (2002). When paying attention becomes counterproductive: impact of divided verses skilled-focused attention on novice and experienced performance of sensoriomotor skills. J. Exp. Psychol. Appl. 8, 6-16.

Bell, J. J., and Hardy, J. (2009). Effects of attentional focus on skilled performance in golf. J. Appl. Sport. Psychol. 21, 163-177.

Bennett, S. (2008). Testing and evaluation; protocols and use, part 1. Strength Cond. J. 30, 39-41.

Castandeda, B., and Gray, R. (2007). Effects of focus of attention on baseball batting performance in players of differing skill levels. J. Sport Exerc. Psychol. 29, 60-77.

Fitts, P. M., and Posner, M. I. (1967). Human Performance. Belmont, CA: Brooks/Cole.

Gabbett, T. J. (2006). Skill-based conditioning games as an alternative to traditional conditioning for rugby league players. J. Strength Cond. Res. 20, 309-315.

Gabbett, T. J., Kelly, J. N., and Sheppard, J. M. (2008). Speed, change of direction speed, and reactive agility of rugby league players. J. Strength Cond. Res. 22, 174-181.

Gentile, A. M. (1972). A working model of skill acquisition with application to teaching. Quest 17, 3-17.

Gentile, A. M. (2000). “Skill acquisition: action, movement, and neuromotor processes," in Movement Science: Foundations for Physical Therapy, 2nd Edn, eds J. H. Carr and R. B.
Shepherd (Rockville, MD: Aspen), 111-187.

Gray, R. (2004).Attending to the execution of a complex sensorimotor skill: expertise differences, choking and slumps. J. Exp. Psychol. Appl. 10, 42-54. Control: Concepts and Applications, 8 th Edn. New York, NY: McGraw-Hill.

Marchant, D. C., Greig, M., and Scott, C. (2009). Attentional focusing instructions influence force production and muscular activity during isokinetic elbow flexions. J. Strength Cond. Res. 23, 2358-2366.

McNevin, N. H., and Wulf, G. (2002). Attentional focus on supra-postural tasks affects postural control. Hum. Mov. Sci. 21, 187-202.

Lawrence, G. P., Gottwald, V.M., Hardy, J., and Khan, M.A. (in press). Internal and external focus of attention in a novice form sport. Res. Q. Exerc. Sport.

Poolton,J.M., Maxwell, J.P., Masters, R.S.W., and Van der Kamp, J. (2007). "Moving with an external focus: autonomic or simply less demanding?" in Wulf on Attentional Focus and Motor Learning [Target article], eds E.-J. Hossner and N.Wenderoth.E-Journal Bewegung und Training 1, 43-44. Available at http:// www.ejournal-but.de

Porter, J. M., Ostrowski, E., Nolan, R., and Wu, F. W. (2010). Standing longjump performance is enhanced when using an external focus of attention. J. Strength Cond. Res. 24, 1746-1750.

Porter, J. M., Wu, W. F. W., and Partridge, J. A. (2010). Focus of attention and verbal instructions: strategies of elite track and field coaches and athletes. Sport Sci. Rev. 19, 199-211.

Raab, M. (2007). "On the value of the attentional focus concept: elaborate and specify!" in Wulf on Attentional Focus and Motor Learning [Target article], eds E.-J. Hossner and N. Wenderoth. E-Journal Bewegung und
Magill, R. A. (2007). Motor Learning and

evaluate performance and measure skill development. The findings presented here lend support in favor of practitioners adopting verbal instructions that induce an external focus of attention; doing so could help patients, students, athletes, clients, or employees achieve success when performing movement-related tasks.

Training 1, 45-46. Available at http:// www.ejournal-but.de

Sayers, A., Sayers, B. E., and Binkley, H. (2008). Preseason fitness testing in national collegiate athletic association. Strength Cond. J. 30, 70-75.

Schücker, L., Hagemann, N., Strauss, B. and Völker, K. (2009). The effect of attentional focus on running economy. J. Sports Sci. 27, 1241-1248.

Spaniol, F. J. (2009). Baseball athletic test: a baseball-specific test battery. Strength Cond. J. 31, 26-29.

Shea, C. H., and Wulf, G. (1999). Enhancing motor learning through external-focus instructions and feedback. Hum. Mov. Sci. 18, 553-571.

Vance, J., Wulf, G., Töllner, T., McNevin, N. H., and Mercer, J. (2004). EMG activity as a function of the performer's focus of attention. J. Mot. Behav. 36, 450-459.

Webb, P., and Lander, J. (1983). An economical fitness testing battery for high school and college rugby teams. Sports Coach 7, 44-46.

Wulf, G. (2007). "Attentional focus and motor learning: a review of 10 years of research," in Gabriele Wulf on Attentional Focus and Motor Learning [Target article], eds E.-J. Hossner and N. Wenderoth. E-Journal Bewegung und Training 1, 4-14. Available at http://www.ejournal-but.de

Wulf, G., Höß, M., and Prinz, W. (1998) Instructions for motor learning: differential effects of internal versus external focus of attention. J. Mot. Behav. 30, 169-179.

Wulf, G., McConnel, N., Gärtner, M., and Schwarz,A. (2002). Enhancing the learning of sport skills through external-focus feedback. J. Mot. Behav. 34, 171-182.

Wulf, G., McNevin, N. H., and Shea, C. H. (2001). The automaticity of complex motor skill learning as a function of attentional focus. Q. J. Exp. Psychol. 54A, 1143-1154.
Wulf, G., and Su, J. (2007). An external focus of attention enhances golf shot accuracy in beginners and experts. Res. Q. Exerc. Sport 78, 384-389.

Wulf, G., Zachry, T., Granados, C., and Dufek, J. S. (2007). Increases in jumpand-reach height through an external focus of attention. Int. J. Sports Sci. Coach. 2, 275-284.

Young, W., and Farrow, D. (2006). A review of agility, practical applications for strength and conditioning. Strength Cond. J. 28, 24-29.

Zachry, T., Wulf, G., Mercer, J., and Bezodis, N. (2005). Increased movement accuracy and reduced EMG activity as the result of adopting an external focus of attention. Brain Res. Bull. 67, 304-309.

Conflict of Interest Statement: The authors declare that the research was conducted in the absence of any commercial or financial relationships that could be construed as a potential conflict of interest.

Received: 05 August 2010; accepted: 14 November 2010; published online: 29 November 2010.

Citation: Porter JM, Nolan RP, Ostrowski EJ and Wulf G (2010) Directing attention externally enhances agility performance: a qualitative and quantitative analysis of the efficacy of using verbal instructions to focus attention. Front. Psychology 1:216. doi: 10.3389/fpsyg.2010.00216

This article was submitted to Frontiers in Movement Science and Sport Psychology, a specialty of Frontiers in Psychology.

Copyright (c) 2010 Porter, Nolan, Ostrowski and Wulf. This is an open-access article subject to an exclusive license agreement between the authors and the Frontiers Research Foundation, which permits unrestricted use, distribution, and reproduction in any medium, provided the original authors and source are credited. 